\title{
The Isomer Effect on Complex Formation in Poly(ethylene oxide)- Nitrophenol System
}

\author{
Kimiaki TsutsuI, Kanato AdachI, Yoshiharu TsustTa, ${ }^{\dagger}$ Hiroaki Yoshimizu, \\ and Takatoshi KinOSHITA
}

Department of Materials Science and Engineering, Polymeric Materials Course, Nagoya Institute of Technology, Gokiso-cho, Showa-ku, Nagoya 466-8555, Japan

(Received May 8, 1998)

\begin{abstract}
Crystalline complex formation of nitrophenol isomers [ $p$-nitrophenol (PNP), $m$-nitrophenol (MNP), and $o$-nitrophenol (ONP)] with poly(ethylene oxide) (PEO) was investigated. The phase diagram in PEO-PNP system obtained by DSC measurement showed a bell-shape region, characteristic of crystalline complex formation, separated by two eutectics. There was only one eutectic in the phase diagram of the PEO-ONP system, which suggested no complex formation. In the PEO-MNP system, there were no peaks in the samples with concentrations from 50 to $70 \mathrm{wt} \%$ of PEO in the DSC thermogram. New peaks in the X-ray diffraction profiles in the PEO-PNP system, were observed in the adduct with $40 \mathrm{wt} \%$ of PEO. In the PEO-ONP system, the only X-ray diffraction peaks assigned to PEO and ONP, respectively, were observed at all concentrations, which showed no complex formation. This might result from intermolecular hydrogen bond formation and self aggregation of ONP molecules. In the PEO-MNP system, amorphous halo increased as a weight fraction of PEO increased, which showed strong interaction of MNP with PEO. The wavenumber of OH stretching of PNP and MNP in the adducts shifted to low wavenumber, but that of ONP did not shift. This shows the hydrogen bond formation of PNP and MNP with PEO. PNP thus forms crystalline complexes with PEO, but not ONP and MNP. Our study suggests that the difference of complex formation among nitrophenol isomers shows that the important factor to form crystalline complexes in PEOnitrophenol system is not only hydrogen bonds between nitrophenol and PEO, but also steric hindrance of nitro group.

KEY WORDS Poly(ethylene oxide) / Nitrophenol Isomer / Crystalline Complex / Hydrogen Bond / Steric Hindrance /
\end{abstract}

Crystalline complexes of polymers with organic compounds have been investigated for separating isomers, preparing highly stereoregular polymers, and obtaining monodispersed fractions from unfractionated polymer samples. ${ }^{1-8}$ In these complexes, the interactions between host and guest molecules are different. Complexes are formed because the external surface of the host polymer presents valleys and ridges, the size and shape of which are such that they can accommodate small molecules. For example, in syndiotactic poly(styrene) (SPS)-organic solvents system, thermoreversible gels are formed by cooling a homogeneous polymer solution below the gelation temperature. ${ }^{9-14}$ The gels consist of crystalline complexes of SPS with organic solvents, housed between cavities generated by two adjacent phenyl groups whose position and distance are given by the helical form: a $2{ }_{1}$ helix. ${ }^{15-17}$ SPS always exhibits the $2_{1}$ helix which consists of TTGG conformation when it complexes with organic solvents because of its stiff chain. Accordingly, it may be expected that organic solvents whose sizes fit the cavities formed by two adjacent phenyl groups of SPS show high ability of complex formation with SPS. ${ }^{18}$

Poly(ethylene oxide) (PEO) forms crystalline complexes either with inorganic compounds such as mercuric chloride ${ }^{19}$ and alkali salts ${ }^{20}$ or with organic compounds such as urea ${ }^{21}$ thiourea, ${ }^{22} p$-dihalogenobenzenes, ${ }^{23}$ and resorcinol. ${ }^{24}$ The conformation of PEO chain as a host is dependent on the complexed guest molecules because of its flexible chain. In the complex of PEO with $p$ dihalogenobenzenes, no specific interactions between host and guest molecules are involved, and the conformation adopted by PEO is nearly the 10/3 helix,

\footnotetext{
† To whom correspondence should be addressed.
}

similar to the $7 / 2$ helix observed for pure PEO. ${ }^{23}$ In the PEO- $p$-nitrophenol (PNP) system, the PEO chain adopts an unusual glide type conformation $\left(\mathrm{T}_{2} \mathrm{GT}_{2} \mathrm{GT}_{3}\right)$ stabilized by hydrogen bonds between the host and guest molecules. ${ }^{25}$ This conformation is quite different from the $7 / 2$ helical conformation of pure PEO.

The size and shape of cavites formed by crystalline polymers that can house small compounds are dependent on the properties of the used polymers (for example, stiffness of the polymer chains). This study reports the isomer effects on complex formation in PEO-nitrophenol system to investigate the possibility of cavities formed by PEO crystal as a recognition site of small compounds. Hydrogen bonds between PEO and nitrophenol isomers and the influence of the steric effect of nitro group on nitrophenol isomers were investigated.

\section{EXPERIMENTAL}

PEO $\left(M_{w}=20000\right)$ were purchased from Wako Pure Chemical Industries Ltd. and used without further purification. Three nitrophenol isomers $(p-, m-$, and $o-$ nitrophenol) (described as PNP, $m$-nitrophenol (MNP), and $o$-nitrophenol (ONP), respectively) were purchased from Nacalai Tesque, Inc. and used without further purification. The samples were prepared by mixing known amounts of PEO with nitrophenol isomers and melting these adducts in an oil bath at the melting temperature of nitrophenol isomers and cooling slowly at room temperature. Differential scanning calorimetry (DSC) thermograms in the range $0-120^{\circ} \mathrm{C}$ were obtained using a Perkin Elmer DSC 7 at a heating rate of $5^{\circ} \mathrm{C} \mathrm{min}^{-1}$ under a nitrogen gas purge in sealed aluminum cells containing about $6 \mathrm{mg}$ of the PEO-nitrophenol 


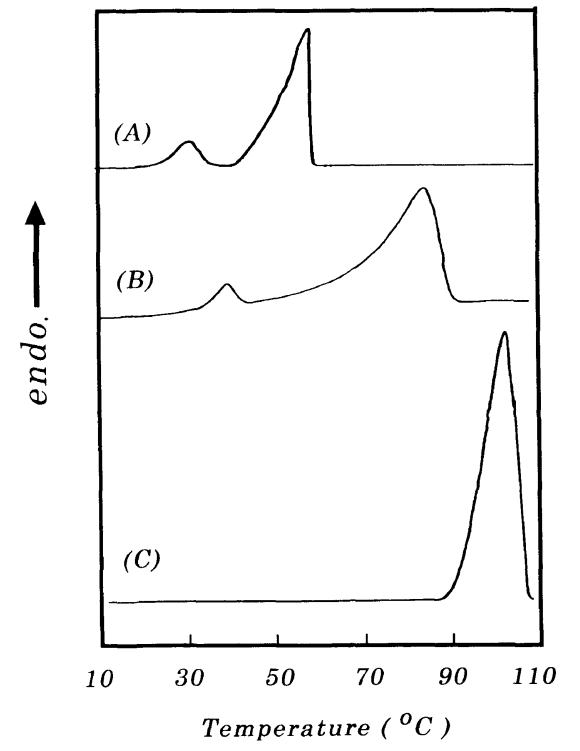

Figure 1. DSC thermograms of the PEO-PNP system: (A) $20 \mathrm{wt} \%$; (B) $50 \mathrm{wt} \%$; (C) $70 \mathrm{wt} \%$ of PNP.

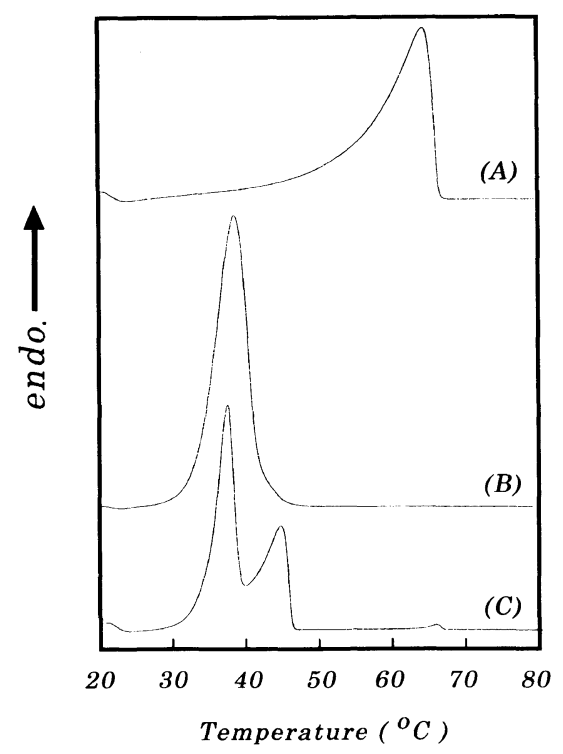

Figure 2. DSC thermograms of the PEO-ONP system: (A) $20 \mathrm{wt} \%$; (B) $50 \mathrm{wt} \%$; (C) $80 \mathrm{wt} \%$ of ONP.

adduct. They were first melted and then recrystallized three times to ensure good homogeneity. Wide-angle $\mathrm{X}$-ray diffraction profiles in the range $3-40^{\circ}$ of $2 \theta$ were recorded with nickel-filtered $\mathrm{Cu}-K_{\alpha}$ radiation by a Rigaku RAD-RC type at the scan rate of $10^{\circ} \mathrm{min}^{-1}$ in the number of 10. Infrared (IR) spectra were obtained using a Perkin Elmer FT-IR spectrometer Spectrum 2000 with the $1 \mathrm{~cm}^{-1}$ resolution in the range $4000-400 \mathrm{~cm}^{-1}$ in the number of 1 .

\section{RESULTS AND DISCUSSION}

\section{Complex Formation of Nitrophenol (NP) Isomers with $P E O$}

DSC measurement was performed on the adducts with various concentrations of $\mathrm{PEO}$ to investigate thermal behavior in the PEO-NP system. Figures $1-3$ show the obtained thermograms of three NP isomers and different thermal behavior among NP isomers. Two peaks are

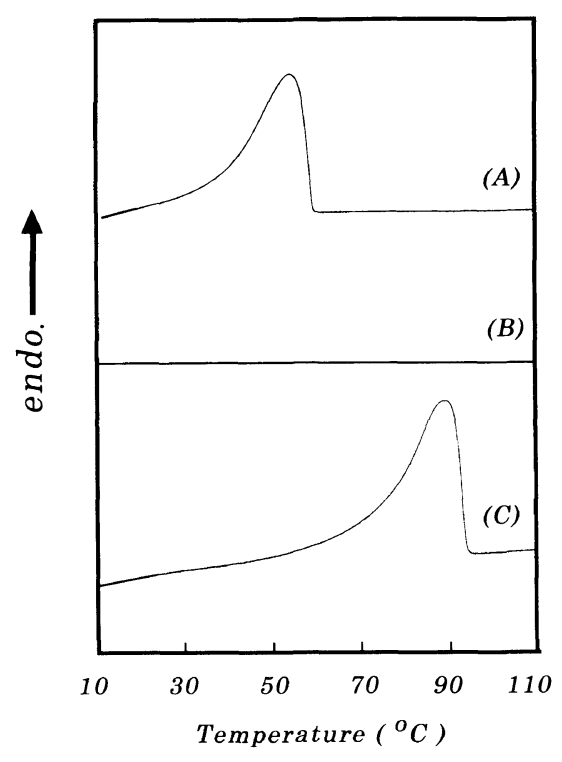

Figure 3. DSC thermograms of the PEO-MNP system: (A) $20 \mathrm{wt} \%$; (B) $50 \mathrm{wt} \%$; (C) $80 \mathrm{wt} \%$ of MNP.

observed for the adducts containing $20 \mathrm{wt} \%$ of PEO in PEO-PNP system. The peaks observed at lower and higher temperature are assigned to the melting of a crystalline complex between PEO and PNP and the melting of pure PEO, respectively. The adducts containing more than $30 \mathrm{wt} \%$ PEO exhibit the melting of PEO at lower temperature and the melting of the complex at higher temperature. There is one peak due to the melting of the complex in the thermogram of the adduct containing $70 \mathrm{wt} \%$ of PEO. These results are consistent with the results obtained by Point and Damman et al. $^{25}$ The samples containing $90 \mathrm{wt} \%$ of PEO exhibit two peaks. The peaks observed at lower temperature and higher temperature are assigned to the melting of PEO and the melting of pure PNP, respectively.

The peaks observed in PEO-ONP system are also dependent on the concentration of PEO, and one or two peaks are observed in the DSC thermograms. In the PEO-MNP system, only one peak is observed at low and high concentration of PEO and no peaks are observed at concentrations from 50 to $80 \mathrm{wt} \%$ of PEO.

The phase diagrams of the adducts in PEO-NP system obtained from DSC thermograms are shown in Figures 4-6. They give supplementary proof of the formation of a crystalline complex between PEO and NP. In general, the phase diagram exhibits two eutectics when a new compound is formed by mixing two different compounds. The phase diagram in PEO-PNP system shows a bell-shape region, characteristic of the crystalline complex, separated by two eutectics as reported. ${ }^{25}$ There is only an eutectic in the phase diagram of PEO-ONP system, observed in the system where a new compound is not formed by mixing two different compounds. This suggests no complex formation in this system. In the PEO-MNP system, there are no peaks in the adducts at concentrations from 50 to $70 \mathrm{wt} \%$, which shows the disappearance of the melting peaks of pure PEO and MNP in this concentration region. The melting enthalpy of PEO $(\Delta H)$ in PEO-ONP and PEO-MNP system is shown in Figure 7 to investigate the influence of the 


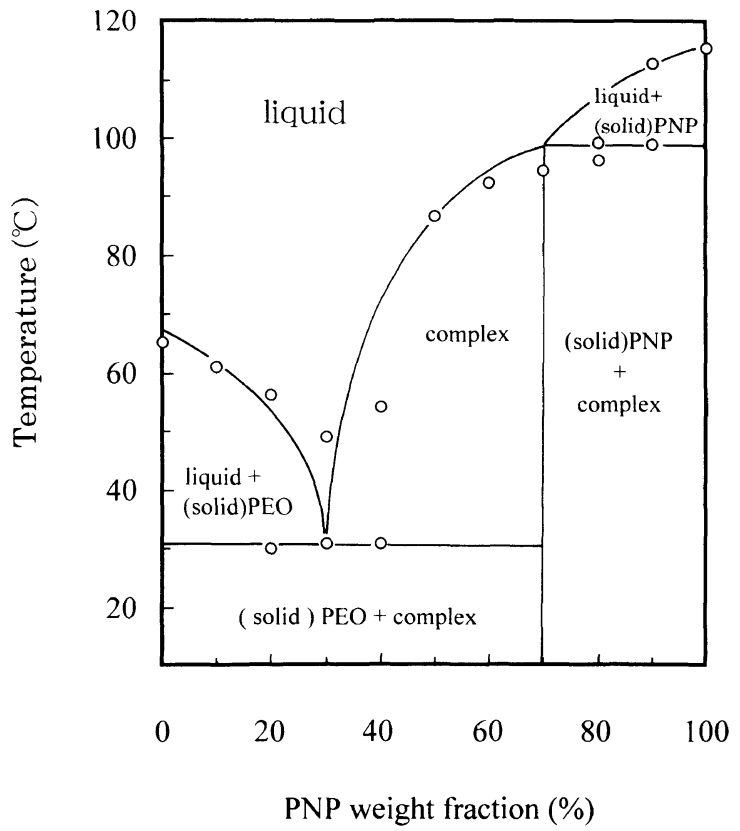

Figure 4. Phase diagram of the PEO-PNP system.

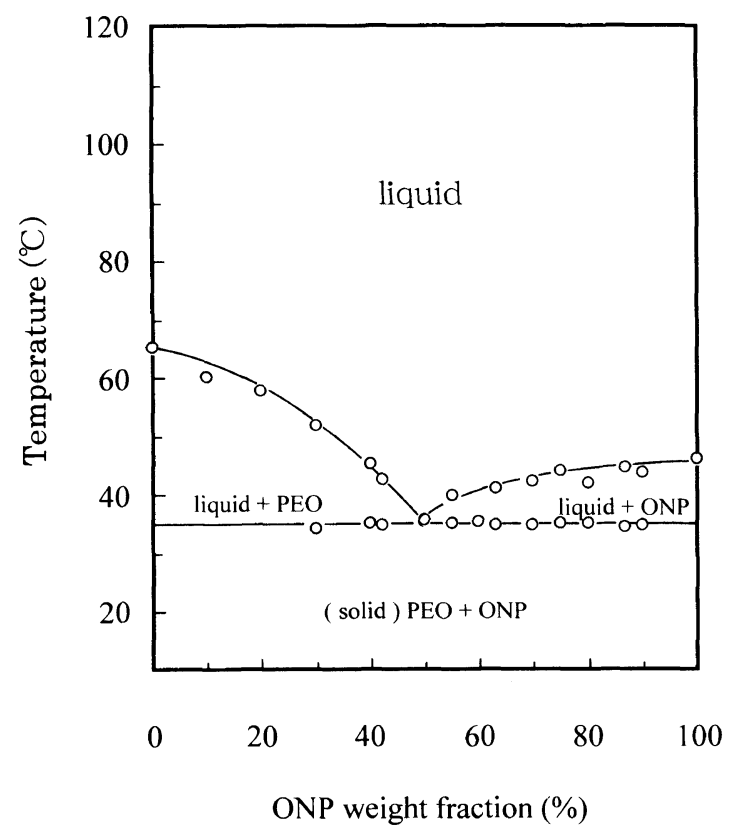

Figure 5. Phase diagram of the PEO-ONP system.

plasticization of ONP and MNP on PEO crystal. $\Delta H$ of PEO component in PEO-MNP system is more largely dependent on a weight fraction of PEO than that of PEO-ONP system. These may result in the strong interaction of MNP with PEO.

$\mathrm{X}$-Ray diffraction measurement was performed on the adducts at various concentrations of PEO to obtain the structural information of the adducts in PEO-NP system. Figures $8-10$ show the obtained X-ray diffraction profiles of the adducts of three NP isomers. In the PEO-PNP system, the intensity of diffraction peaks of PEO decreases as the concentration of PEO increases, and new peaks are observed in the profiles of the samples with $40 \mathrm{wt} \%$ of PEO (denoted by arrow in Figure 8 ). The new peaks are assigned to the crystalline complex of PEO with PNP and this is consistent with Point and Damman et al. ${ }^{25}$ In the PEO-ONP system, the only

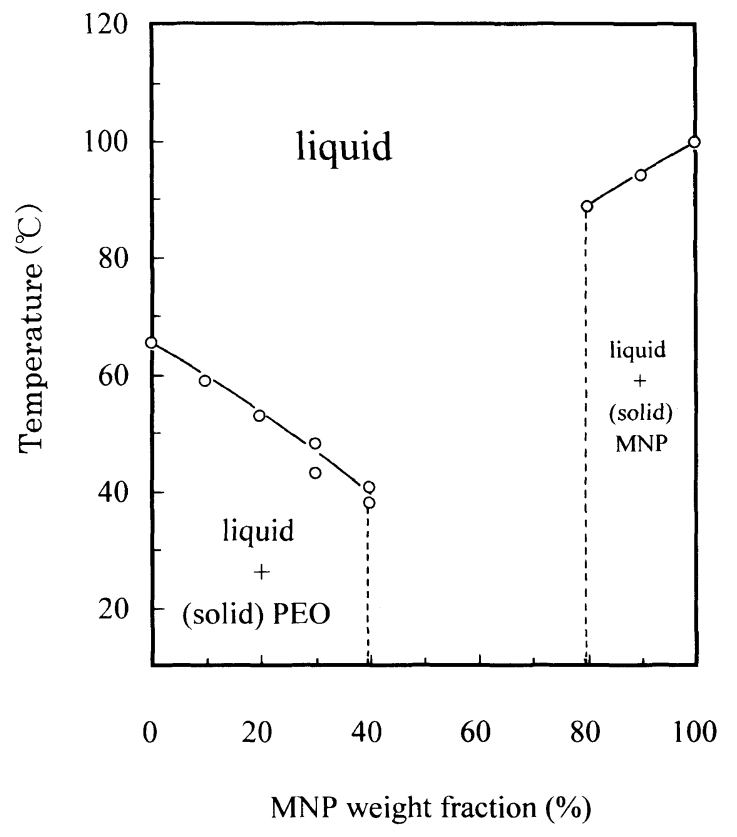

Figure 6. Phase diagram of the PEO-MNP system.

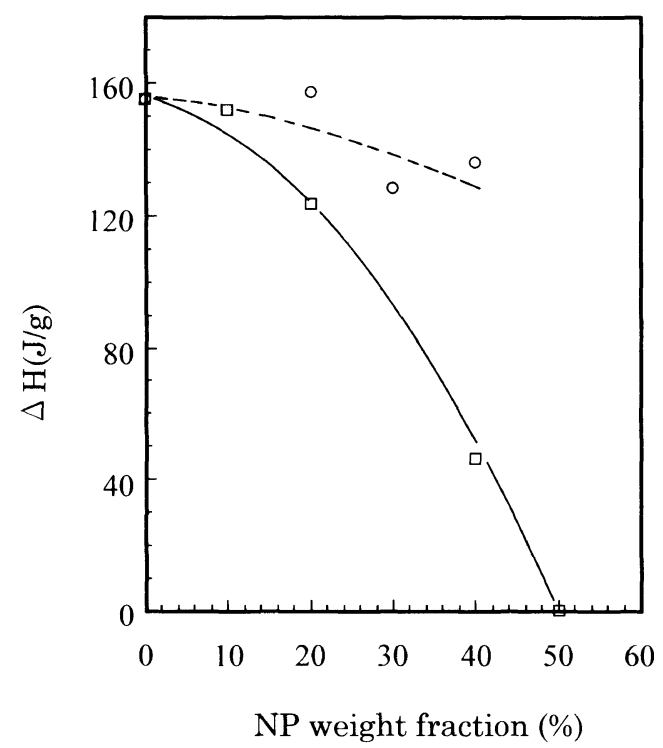

Figure 7. Melting enthalpy $(\Delta H)$ of PEO in the PEO-ONP $(O)$ and PEO-MNP ( $\square$ ) system.

peaks assigned to pure PEO and ONP are observed in the whole concentration although the intensity of the peaks changes in proportion to a weight fraction of PEO, which shows no complex formation. This may be because ONP forms intermolecular hydrogen bonds and self aggregation of ONP molecules occurs without interaction with PEO. In the PEO-MNP system, it is observed that the amorphous halo increases as the weight fraction of PEO increases. Only $20 \mathrm{wt} \%$ of MNP can plasticize PEO and distort PEO crystals, which shows the strong interaction of MNP with PEO, which is consistent with the thermal behavior of the adducts in PEO-MNP system. That the adducts in PEO-MNP system is the highest fluidity of the adducts in PEO-NP system may support the strong interaction between PEO and MNP.

PEO forms a crystalline complex with PNP, but not form with ONP or MNP judging from the phase diagrams 


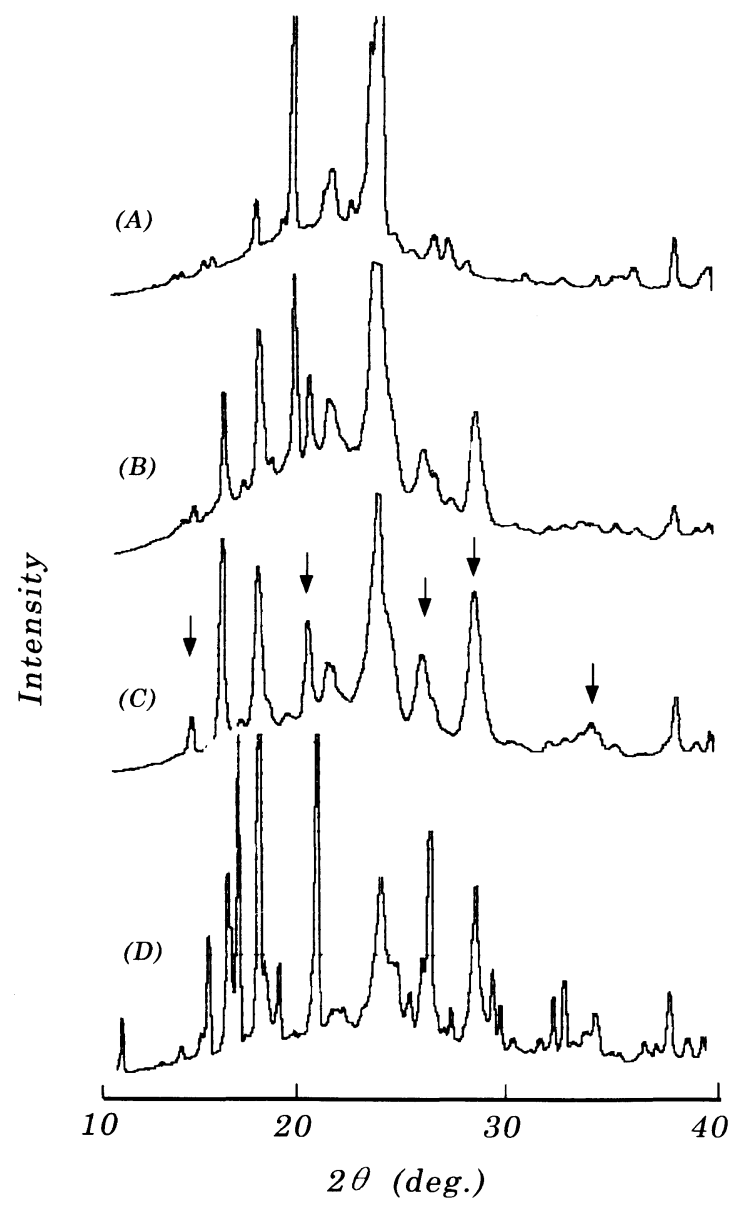

Figure 8. X-Ray diffraction profiles of the PEO-PNP system: (A) $20 \mathrm{wt} \%$; (B) $40 \mathrm{wt} \%$; (C) $60 \mathrm{wt} \%$; (D) $80 \mathrm{wt} \%$ of PNP.

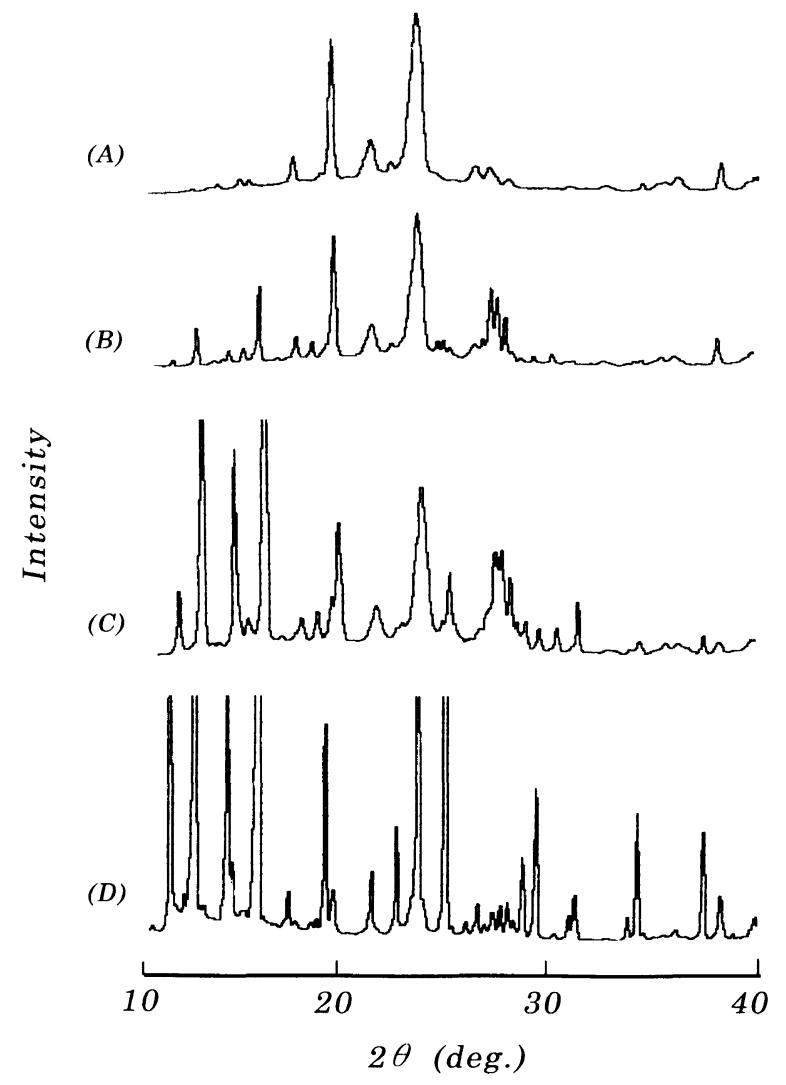

Figure 9. X-Ray diffraction profiles of the PEO-ONP system: (A) $20 \mathrm{wt} \%$; (B) $40 \mathrm{wt} \%$; (C) $60 \mathrm{wt} \%$; (D) $80 \mathrm{wt} \%$ of ONP.

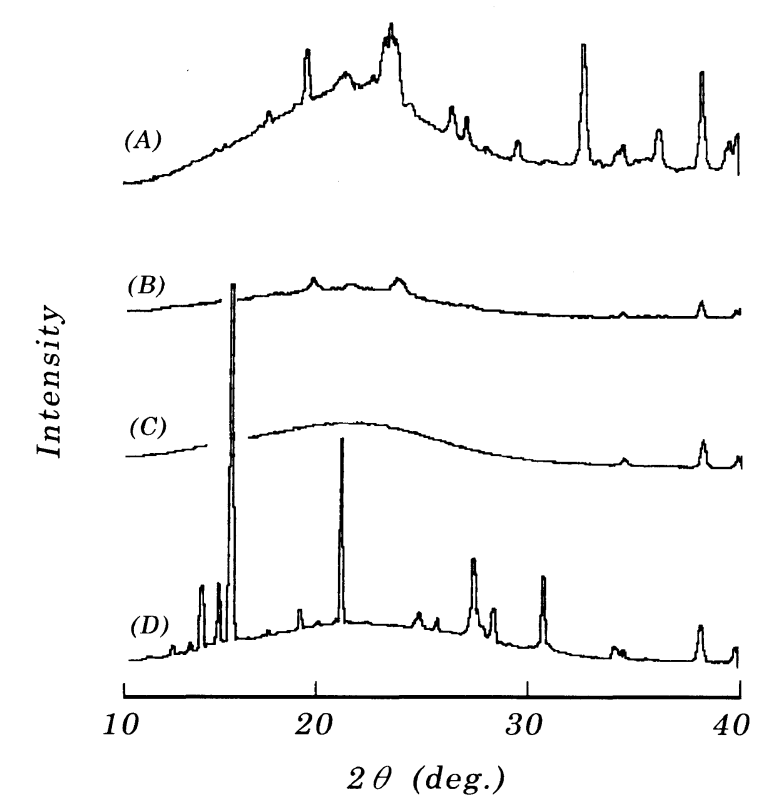

Figure 10. X-Ray diffraction profiles of the PEO-MNP system: (A) $20 \mathrm{wt} \%$; (B) $40 \mathrm{wt} \%$; (C) $60 \mathrm{wt} \%$; (D) $80 \mathrm{wt} \%$ of MNP.

and X-Ray diffraction profiles of the PEO-NP system. MNP has large influence on PEO. The size and shape of complexed molecules are very important factors to determine the ability of complex formation. But PEO forms crystalline complexes with various compounds thus changing the conformation of its chain, dependent on the complexed molecules because of its flexible chains. For example, in the case of no specific interactions between host and guest molecules, PEO can form crystalline complexes with $p$-dihalogenobenzenes with different molecular sizes. ${ }^{23}$ The thermal behavior of the adducts in PEO-NP system shows interactions between PEO and NP isomers are different, and the complex formation is strongly dependent on NP isomers, which shows the possibility of cavities formed by PEO crystal as a recognition site of small compounds. We consider the ability of hydrogen bonds on NP with PEO as a factor of complex formation from thermal behavior and X-ray diffraction profiles in PEO-NP system.

\section{Influence of Hydrogen Bonds on Complex Formation in PEO-NP System}

Three NP isomers possess a hydroxyl group $(\mathrm{OH})$ and should form hydrogen bonds between the hydroxyl group and oxygen atom of PEO. PNP guest molecules are capable of forming hydrogen bonds with PEO and cause large modification of the conformation of the PEO chain. ${ }^{26}$ So hydrogen bonds between PEO and NP as interactions are very important to complex formation. The wavenumber of $\mathrm{OH}$ stretching shifts to low wavenumber by intra- and intermolecular interaction (hydrogen bond) in IR spectroscopy. IR spectra in the PEOPNP system are shown in Figure 11. The wavenumber of the $\mathrm{OH}$ stretching is $3326 \mathrm{~cm}^{-1}$ in the crystalline PNP and $3271 \mathrm{~cm}^{-1}$ in the complex as reported in the literature. ${ }^{26} \mathrm{~A}$ comparison of the wavenumber of $\mathrm{OH}$ stretching of three NP isomers in the pure crystalline and adducts is summarized in Table I. The wavenumbers of $\mathrm{OH}$ stretching of PNP and MNP in the adducts shift to low wavenumber. These show the hydro- 


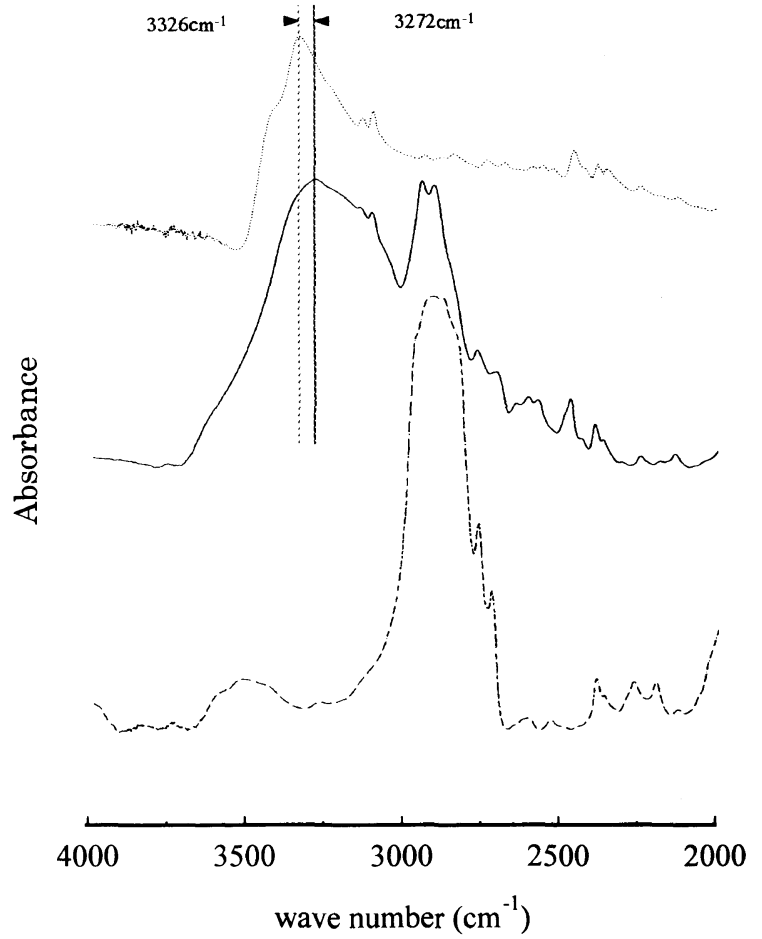

Figure 11. IR spectra of (A) pure PNP, (B) $60 \mathrm{wt} \%$ of PNP, and (C) pure PEO.

Table I. Frequencies of $\mathrm{OH}$ stretching for nitrophenol isomers in the PEO-NP system

\begin{tabular}{lcc}
\hline Sample & Pure $/ \mathrm{cm}^{-1}$ & Mixed $/ \mathrm{cm}^{-1}$ \\
\hline ONP & 3438 & 3437 \\
MNP & 3389 & 3204 \\
PNP & 3326 & 3272 \\
\hline
\end{tabular}

gen bond formation of PNP or MNP with PEO. The wavenumber of $\mathrm{OH}$ stretching of ONP in the adducts does not shift to low wavenumbers, which results in no hydrogen bond formation of ONP with PEO. No hydrogen bond formation between PEO and ONP is due to the intramolecular hydrogen bond formation and self-aggregation of ONP molecules.

From DSC and X-ray measurements only PNP can form a crystalline complex with PEO, but not MNP and ONP. No complex formation of OMP which cannot form hydrogen bonds with PEO shows that hydrogen bond formation of NP with PEO is an important factor. MNP molecules can form hydrogen bonds with PEO and plasticize PEO chain as mentioned above, but cannot form a crystalline complex with PEO. The difference of complex formation between PNP and MNP may suggest that the important factor to form crystalline complexes in PEO-NP system is not only hydrogen bonds between $\mathrm{OH}$ of NP and oxygen atom of PEO, but also steric hindrance of the nitro group of NP.

PEO forms crystalline complexes with various compounds changing the conformation of its chain, dependent on the complexed molecules in the case of no specific interactions between host and guest molecules. But when there are specific interactions between host and guest molecules in the system such as PEO-NP system, by considering these obtained results, factors that determine the ability of complex formation among NP isomers are (1) hydrogen bonds of NP with PEO and (2) steric hindrance of nitro group of NP and only PNP molecules which satisfies both conditions to form a crystalline complex with PEO. These results show the possibility of cavities formation by PEO crystals as recognition sites of NP isomers.

\section{CONCLUSIONS}

PEO forms crystalline complexes with various small compounds because of its flexible chain, but PEO exhibits different dependence of complex formation on nitrophenol isomers. Only PNP forms crystalline complexes with PEO as shown from DSC and X-ray measurements. Investigation of hydrogen bonds between PEO and NP isomers by IR spectroscopy shows that the wavenumber of OH stretching of PNP and MNP in the adducts shifts to low wavenumbers, but that of ONP does not shift. Factors for the ability of complex formation with PEO among NP isomers would thus appear to be (1) hydrogen bonds of NP with PEO and (2) steric hindrance of nitro group of NP and only PNP which satisfied both conditions to form crystalline complexes with PEO.

Thus, our study shows that PEO recognizes NP isomers and forms a crystalline complex with only PNP. We suggest the possibility of cavities formed by PEO crystal as recognition sites of small compounds.

\section{REFERENCES}

1. W. T. Winter and A. Sarko, Biopolymers, 13, 1461 (1974).

2. H. Kusuyama, N. Miyamoto, Y. Chatani, and H. Tadokoro, Polym. Commun., 24, 119 (1983).

3. J. Watanabe, Y. Sasanuma, A. Endo, and I. Uematsu, Polymer, 25, 698 (1984).

4. Y. Chatani and T. Irie, Polymer, 29, 2126 (1988).

5. Y. Chatani, Y. Shimane, and T. Inagaki, Polymer, 34, 1620 (1993)

6. A. E. Tonelli, Polymer, 35, 573 (1994).

7. A. Kawaguchi, Polymer, 35, 3797 (1994).

8. N. Vasanthan, I. D. Shin, and A. E. Tonelli, Macromolecules, 29, 263 (1996).

9. F. Deberdt and H. Berghmans, Polymer, 34, 2192 (1993).

10. M. Kobayashi and T. Kozasa, Appl. Spectrosc., 47, 1417 (1993).

11. F. Deberdt and H. Berghmans, Polymer, 35, 1694 (1994).

12. C. Daniel, C. Dammer, and J.-M. Guenet, Polymer, 35, 4243 (1994).

13. T. Roels, F. Deberdt, and H. Berghmans, Macromolecules, 27, 6216 (1994).

14. T. Roels, F. Deberdt, and H. Berghmans, Progr. Colloid Polym. Sci., 102, 82 (1996)

15. C. Daniel, M. D. Deluca, J.-M. Guenet, A. Brulet, and A. Menelle, Polymer, 37, 1273 (1996).

16. J.-M. Guenet, TRIP, 4, 6 (1996).

17. C. Damiel, A. Memelle, A. Brulet, and J.-M. Guenet, Polymer, 38, 4193 (1997).

18. K. Tsutsui, H. Fukatsu, T. Katsumata, Y. Tsujita, H. Yoshimizu, and T. Kinoshita, submitted to Polymer.

19. R. Iwamoto, Y. Saito, H. Ishihara, and H. Tadokoro, J. Polym. Sci., Part A-2, 6, 1509 (1968).

20. Y. Chatani and S. Okamura, Polymer, 28, 1815 (1987).

21. I. Tadokoro, T. Yoshihara, Y. Chatani, and S. Murahashi, Polym. Lett., 2, 369 (1964).

22. J. Parrod and A. Kohler, Compt. Rend., 245, 1046 (1958).

23. J. J. Point and C. Coutelier, J. Polym. Sci., Polym. Phys. Ed., 23, 231 (1985).

24. E. Delaite, J. J. Point, P. Damman, and M. Dosiere, Macromolecules, 25, 4768 (1992).

25. J. J. Point and P. Damman, Macromolecules, 25, 1184 (1992).

26. P. Damman and J. J. Point, Macromolecules, 26, 1722 (1993) 